\title{
ANALYTICAL AND NUMERICAL INVESTIGATION FOR THREE DIMENSIONAL HEAT PROBLEMS IN AN ANISOTROPIC MEDIUM WITH INTERNAL HEAT SOURCES
}

Andreas MANDELis ${ }^{1}$, KARL-Heinz GressleHneR ${ }^{2}$, GÜNTHER HENDORFER ${ }^{2}$

Ontario, Canada $\quad{ }^{2}$ Upper Austrian University of Applied Sciences, Research \& DeVelopment LtD., 4600 Wels, Austria

\section{INTRODUCTION}

Many materials of industrial interest do not conduct heat equally well in all directions and are called anisotropic bodies. This holds for example for crystals, wood, laminates, fiber-reinforced composites, and many other materials. Therefore, heat conduction in anisotropic materials has numerous important applications in various branches of science and engineering. In this work we present an analytical solution of the three dimensiononal heat conduction equation in anisotropic media.

\section{THEORY}

The geometry of the structure investigated is shown in Fig. (1), with homogeneous boundary conditions of the third kind.

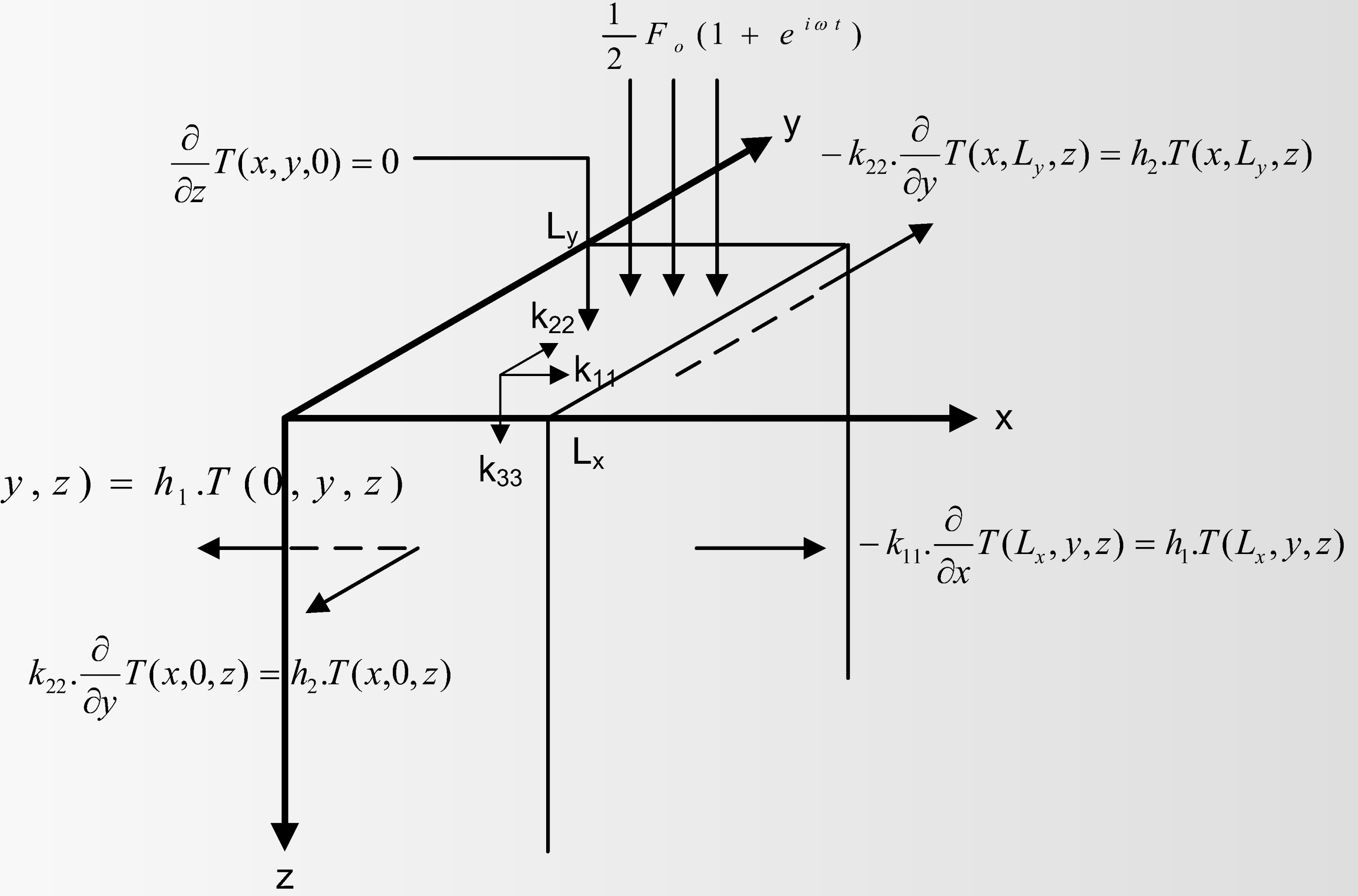

Figure 1: Right semi-infinite slab geometry in Cartesian coordinates with mixed homogeneous boundary conditions on five surfaces. The amplitude of the thermal-wave heat flux is uniform distributed across the surface $z=0$.

The governing partial differential equation is given by

$$
\begin{gathered}
\rho c \frac{\partial}{\partial t} T(x, y, z ; t)=k_{11} \frac{\partial^{2} T}{\partial x^{2}}+k_{22} \frac{\partial^{2} T}{\partial y^{2}}+k_{33} \frac{\partial^{2} T}{\partial z^{2}}+\left(k_{12}+k_{21}\right) \frac{\partial^{2} T}{\partial x \partial y} \\
+\left(k_{23}+k_{32}\right) \frac{\partial^{2} T}{\partial y \partial z}+\left(k_{31}+k_{13}\right) \frac{\partial^{2} T}{\partial z \partial x}+Q(x, y, z ; t)
\end{gathered}
$$

where $k_{i j}$ is the thermal conductivity tensor $(i=1,2,3$ and $j=1,2,3)$.

To eliminate cross-derivatives in Eq. (1) a special linear coordinate transform is introduced which reduces Eq. (1) into a canonical form (Eq. (2)) [2].

$$
\begin{aligned}
& X=x+q_{1} y+q_{2} z \\
& Y=q_{3} y+q_{4} z \\
& Z=q_{5} z
\end{aligned}
$$

$q_{1}$ to $q_{5}$ in Eq. (2) are the coordinate transformation coefficients.

For orthotropic media Eq. (1) reduces to

$$
\nabla^{2} T(X, Y, Z ; \omega)-\sigma_{e}^{2} T(X, Y, Z ; \omega)=-\frac{Q(X, Y, Z ; \omega)}{k_{e}}
$$

with $q_{1}=q_{2}=q_{4}=0, q_{3}=\sqrt{k_{11} / k_{22}}, q_{5}=\sqrt{k_{11} / k_{33}}, \alpha_{e}=q_{5}^{2} \alpha_{33}, k_{e}=q_{5}^{2} k_{33}$ and $\sigma_{e}=\sqrt{i \omega / \alpha_{e}}$. $\alpha_{e}$ is the effective thermal diffusivity, $k_{e}$ is the effective thermal conductivity and $\sigma_{e}$ is the dipsersive complex wave-number.

\section{GREEN'S FUNCTION}

The boundary value problem was solved by derivation and use of frequency-domain Green's function [3]

$$
\begin{array}{r}
\nabla^{2} G\left(X, Y, Z \mid X_{0}, Y_{0}, Z_{0} ; \omega\right)-\sigma_{e}^{2} G\left(X, Y, Z \mid X_{0}, Y_{0}, Z_{0} ; \omega\right) \\
=-\frac{1}{\alpha_{e}} \delta\left(X-X_{0}\right) \delta\left(Y-Y_{0}\right) \delta\left(Z-Z_{0}\right)
\end{array}
$$

The application of the boundary conditions leads to eigenvalue equations and after some algebra to the desired Green's function [3].

$$
\begin{aligned}
G\left(X, Y, Z, \mid X_{0}, Y_{0}, Z_{0} ; \omega\right) & =\frac{1}{2 \cdot \alpha_{e}} \sum_{l=1}^{\infty} \sum_{m=1}^{\infty} A_{l_{m}} \Phi_{l}(X) \Psi_{m}(Y) \\
& \times\left[e^{-c_{l_{m}}\left|Z-Z_{0}\right|}+e^{-c_{l_{m}}\left(Z+Z_{0}\right)}\right] \quad 0 \leq Z<\infty
\end{aligned}
$$

The series expansion coefficient $A_{l_{m}}$ is given by Eq. (6a) and the complex thermal wave number $c_{l_{m}}$ associated with the $(l, m)$-mode is given by Eq. $(6 \mathrm{~b})$

$$
A_{l_{m}}=\frac{\Phi_{l}\left(X_{0}\right) \Psi_{m}\left(Y_{0}\right)}{\int_{0}^{L_{x}} \Phi_{l}{ }^{2}\left(X^{\prime}\right) \mathrm{d} X^{\prime} \int_{0}^{q_{3} L_{y}} \Psi_{m}{ }^{2}\left(Y^{\prime}\right) \mathrm{d} Y^{\prime}}
$$$$
{ }_{l_{m}}^{2}(\omega)=\beta_{l}^{2}+\gamma_{m}^{2}+\sigma_{e}^{2}(\omega)
$$

(6b)

$\Phi_{l}(X)$ and $\Psi_{m}(Y)$ in Eq. (5) are the eigenfunctions.

\section{ACKNOWLEDGEMENTS}

This work was financially supported by the TAKE OFF program of the Bundesministerium für Verkehr, Innovation und Technologie (BMVIT). Furthermore, we wish to thank our cooperation partners FACC AG and Secar Technology GmbH.

Andreas Mandelis gratefully acknowledges the NSERC Strategic Grant STPGP 430440 for non-destructive imaging of manufacturing flaws.

\section{THERMAL-WAVE FIELD}

For thermographic (lock-in) imaging a uniform harmonically modulated thermal-wave flux across the plane $Z=z=0$ was assumed (Eq. (7))

\begin{tabular}{|c|c|c|c|c|c|c|c|c|c|}
\hline Material & $\begin{array}{c}k_{11} \\
\frac{\mathrm{W}}{(\mathrm{m} \mathrm{K})}\end{array}$ & $\begin{array}{l}k_{22} \\
\mathrm{~W} \\
(\mathrm{~m} \mathrm{~K})\end{array}$ & $\begin{array}{c}k_{33} \\
\mathrm{~W} \\
(\mathrm{~m} \mathrm{~K})\end{array}$ & $\begin{array}{c}\rho \\
\frac{\mathrm{kg}}{\left(\mathrm{m}^{3}\right)}\end{array}$ & $\begin{array}{c}c \\
\frac{\mathrm{J}}{(\mathrm{kg} \mathrm{K})}\end{array}$ & $\begin{array}{c}c h_{1} \\
\mathrm{~W} \\
\left(\mathrm{~m}^{2} \mathrm{~K}\right)\end{array}$ & $\begin{array}{c}c h_{2} \\
\mathrm{~W} \\
\left(\mathrm{~m}^{2} \mathrm{~K}\right)\end{array}$ & $\begin{array}{l}\alpha_{e} \\
\frac{m^{2}}{\mathrm{~s}}\end{array}$ & $\begin{array}{c}k_{e} \\
\mathrm{~W} \\
(\mathrm{mK})\end{array}$ \\
\hline anisotropic & 7 & 0.7 & 0.7 & 1490 & 1200 & 13 & 13 & $3.91 E-6$ & 7 \\
\hline
\end{tabular}

$$
F(X, Y ; \omega)=\left\{\begin{array}{cl}
\frac{1}{2} F_{0}\left(1+e^{i \omega t}\right) & 0 \leq X \leq L_{x} \text { and } 0 \leq Y \leq q_{3} L_{y} \\
0 & \text { otherwise }
\end{array}\right.
$$

$F_{0}$ is the amplitude and $\omega$ is the modulation frequency of the incident thermal-wave flux. The thermal-wave field is given by

$$
T(X, Y, Z ; \omega)=\frac{F_{0}}{2\left(k_{11} k_{33}\right)^{1 / 2}} \sum_{l=1}^{\infty} \sum_{m=1}^{\infty} B_{l_{m}} \Phi_{l}(X) \Psi_{m}(Y) e^{-c_{l_{m}} Z}
$$

with the series expansion coefficient

$$
B_{l_{m}}=\frac{\int_{0}^{L_{x}} \Phi_{l}\left(X_{0}\right) \mathrm{d} X_{0} \int_{0}^{q_{3} L_{y}} \Psi_{m}\left(Y_{0}\right) \mathrm{d} Y_{0}}{\int_{0}^{L_{x}} \Phi_{l}^{2}\left(X^{\prime}\right) \mathrm{d} X^{\prime} \int_{0}^{q_{3} L_{y}} \Psi_{m}{ }^{2}\left(Y^{\prime}\right) \mathrm{d} Y^{\prime}}
$$

\section{COMPUTER MODELING OF THE ANALYTICAL SOLUTION}

The parameters used for simulation are given in Tab. 1.

The Green function (Eq. 5) and the thermal-wave field (Eq. 8) for a uniform incident thermalave flux were computed with the mathematical software MAPLE.
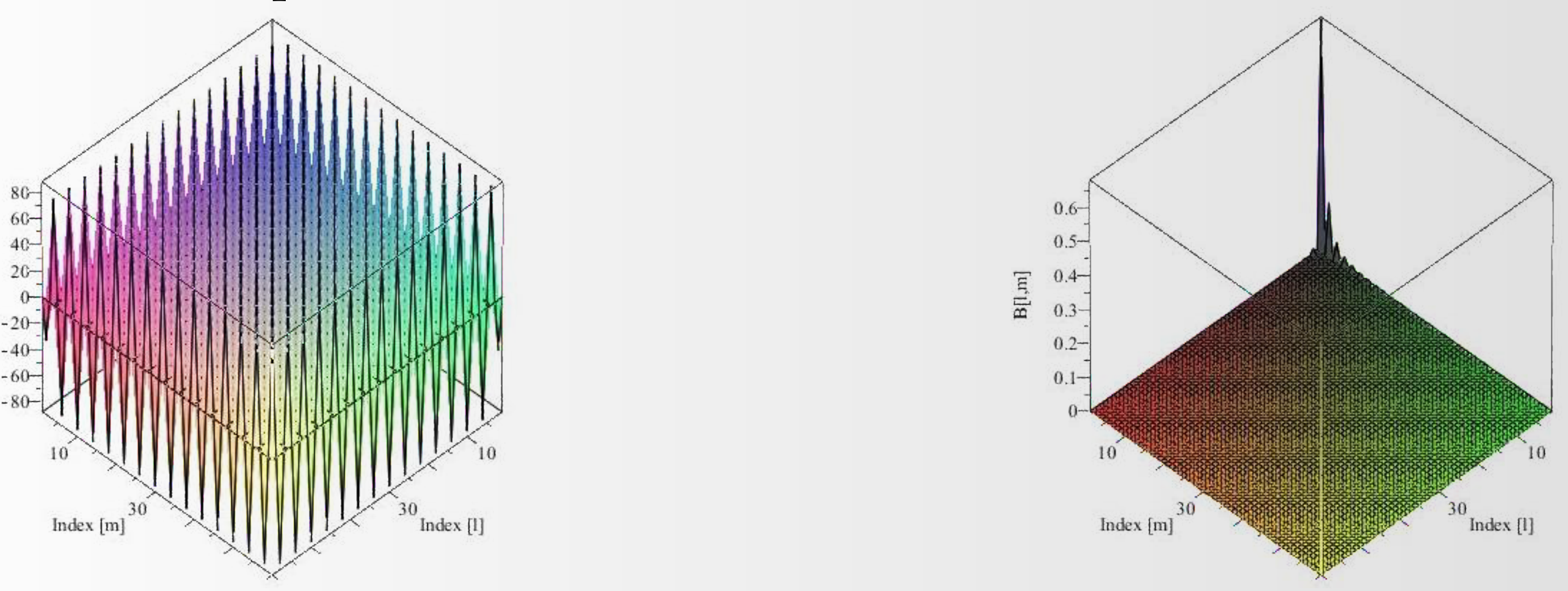

Figure 2: Series expansion coefficients of a) the Green's function $A_{l_{m}}$ and b) the thermal-wave field $B_{l_{m}}$ of an anisotropic material. The oscillating behaviour of the $A_{l_{m}}$ is predominant. The convergence behaviour of $B_{l_{m}}$ is rather poor (usually appr. more than 100 elements must be taken into account).
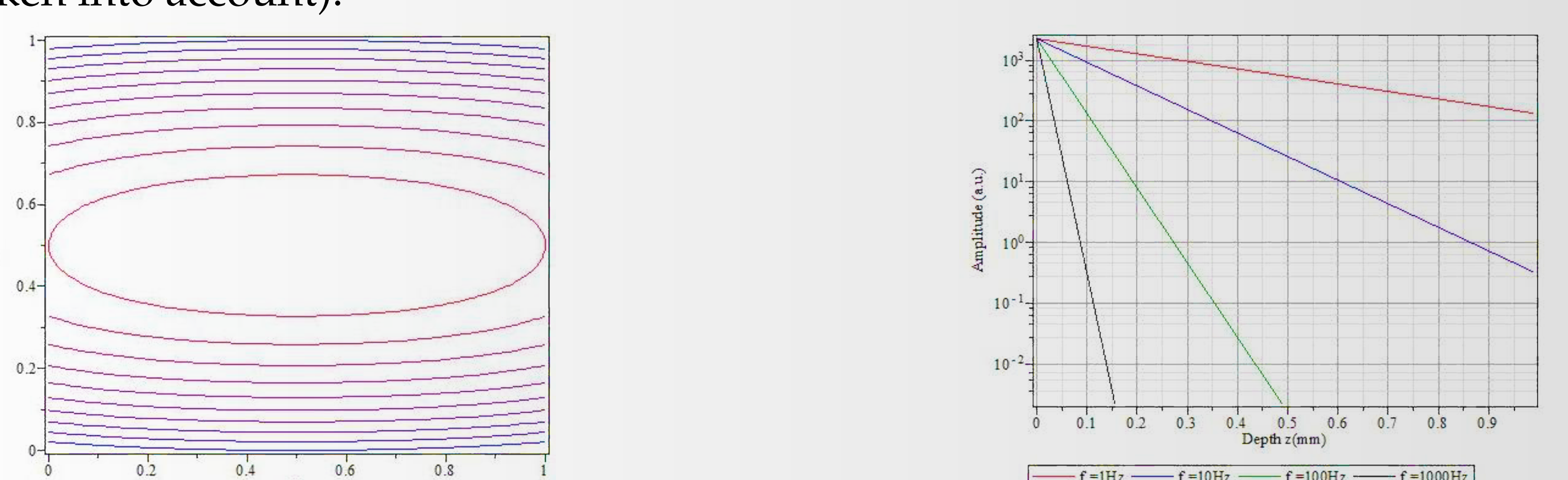

Figure 3: a) Contour plot of the Green's function in the normalized $x-y$-plane for $z=0$ b) Amplitude of the thermal-wave field vs. depth $z$ with frequency as a parameter.

EXPERIMENT AND SIMULATION WITH Finite ELEMENT METHOD To demonstrate the influence of different thermal conductivities in $\mathrm{x}$ - and $\mathrm{y}$-direction, the temperature distribution of an unidirectional CFRP (Carbon Fiber Reinforced Plastic) sample was investigated in the plane $z=0$. In this case, the incident heat flux is a point like, pulsed laserbeam source instead of a modulated, uniform thermal-wave flux given in Eq. (7). During the measurement the $x$-axis was orientated along the higher thermal conductivity of the CFRP sample $\left(k_{11}\right.$ in Table 1$)$. However, the experimental results and the results of FEM simulation are only qualitative. That means, in both cases the temperature distributions are represented in arbitrary units (cold to hot)

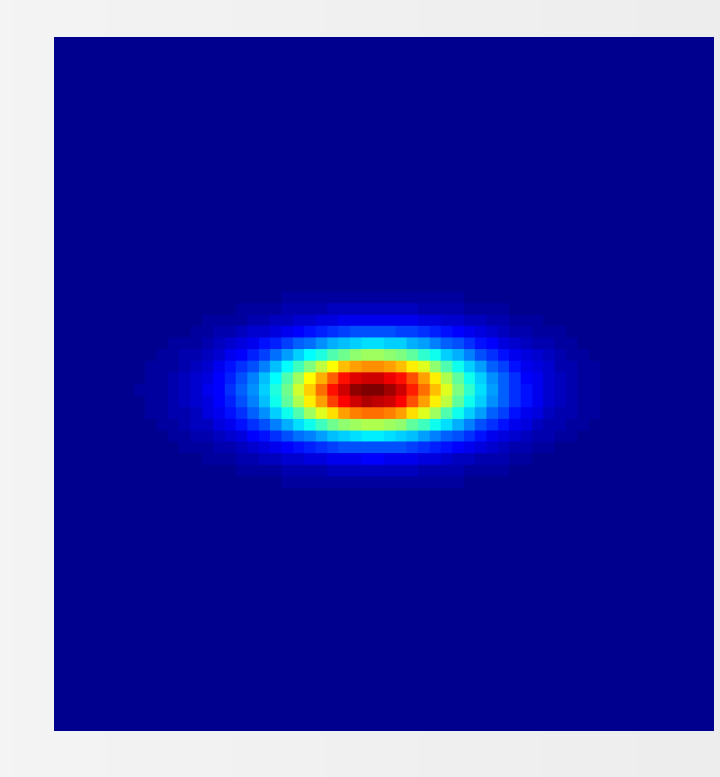

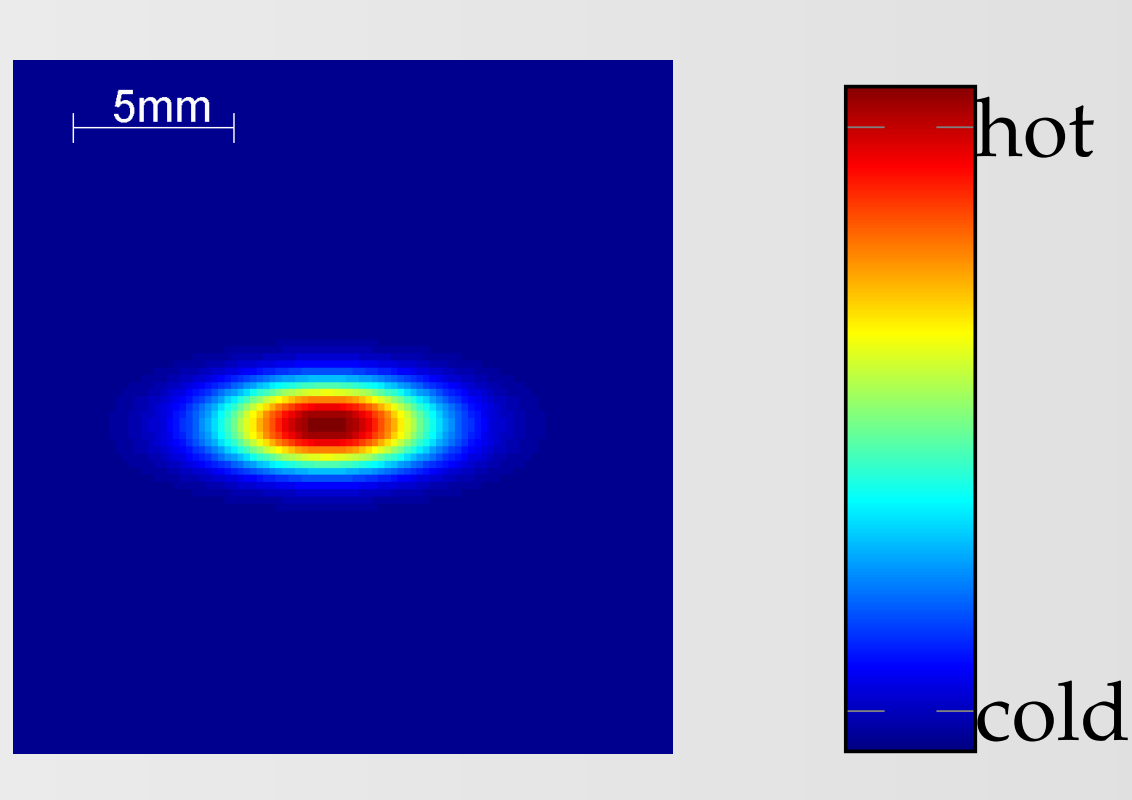

Figure 3: a) Experimental investigation of the unidirectional CFRP sample with the following parameters: spot size of the laser-beam: appr. $0.1 \mathrm{~mm}$, pulse duration of the laser-beam: appr.0.5 sec, sampling frequency: $50 \mathrm{~Hz}$, observation time: $10 \mathrm{sec}$ after excitation. b) Results of simulation with FEM. In order to take into account the experimental conditions, the boundary condition at $z=0$ is not the same as shown in Fig. 1 and is given by: $-k_{3} \frac{\partial}{\partial} T(x, y, 0)=q_{0}-h_{3} T(x, y, 0)$. $q_{0}$ is the . -direction and was choosen equal to $h_{1}$.

\section{REFERENCES}

[1] K.D. Cole. Heat Conduction Using Green's Functions. Series in computational and physical processes in mechanics and thermal sciences. Taylor \& Francis Group, 2010.

[2] Ming-Hsien Hsieh and Chien-Ching Ma. Analytical investigations for heat conduction problems in anisotropic thin-layer media with embedded heat sources. International Journal of Heat and Mass Transfer, 45(20):4117 - 4132, 2002.

[3] A. Mandelis. Diffusion-Wave Fields: Mathematical Methods and Green Functions. Springer, 2001 [4] N. Ozisik. Heat Conduction. A Wiley-Interscience publication. Wiley, 1993. 